\title{
Dynamics of indistinguishable free particles driven by a quantum bath
}

\author{
Marco Nizama ${ }^{1, *}$ and Manuel O. Cáceres ${ }^{2, \dagger}$ \\ ${ }^{1}$ Departamento de Física, Universidad Nacional del Comahue and CONICET, 8300 Neuquén, Argentina \\ ${ }^{2}$ Centro Atómico Bariloche, CNEA, Instituto Balseiro and CONICET, 8400 Bariloche, Argentina
}

(Received 14 February 2019; published 8 April 2019)

\begin{abstract}
The time evolution of correlations for two indistinguishable free particles, in a one-dimensional lattice interacting with a quantum bath, is studied in the Born-Markov approximation. The analytical solution for the density matrix is obtained, for bosons and fermions, in the second quantization formalism. The time evolution of the negativity of indistinguishable particles is analyzed for quantum mixed states. Quantum correlations heavily depend on the selected bipartition; in return, a small difference between the statistics of the particles is noted, due to the Pauli exclusion principle. In the presence of dissipation, the negativity (a nonclassical correlation) shows a time-oscillatory behavior for a particular geometrical bipartition. The total probability of finding one particle in each subsystem is also calculated and shows consistent behavior.
\end{abstract}

DOI: 10.1103/PhysRevA.99.042107

\section{INTRODUCTION}

It has been pointed out that quantum systems that are not directly coupled to each other may experience a complex dynamics due to indirect bath-mediated coupling [1]. These results emphasized the fact that taking into account bath degrees of freedom will improve the coherence description of any particular microscopic system [2]. The problem mentioned is somewhat reminiscent of the study of fluctuations and dissipation in a classical system [3], but the extension of this task to the quantum world still has unknown features. One possible way to get insight is the construction of a suitable generalized infinitesimal generator [2-5]. Cases where bathmediated inference has been reported, for instance, can be found in synchronization of open spin systems [6], energy transfer in photosynthetic systems [7], quantum spin chains immersed in a common thermal bath [8], and entanglement in a system of two (two-level) atoms interacting with a squeezed vacuum [9].

As a prototypical model to study the exact solution of an open quantum system, we will focus our attention on a system of free indistinguishable particles interacting with a common quantum thermal bath. Then, as the system $\mathcal{S}$ we consider two free tight-binding particles in a one-dimensional lattice interacting (linearly) with a phonon bath $\mathcal{B}$. The interaction with $\mathcal{B}$ will be mediated by shift (nearest-neighbor translational) operators acting on the particles. To describe these shift operators, we use the second quantization formalism. Previous experiences with these types of systems have been introduced using distinguishable particles [10,11]. These free models are many-body extensions of the well-known dissipative quantum-walk approach [12-17], which has been shown to be very suitable for describing the superposition of quantum

\footnotetext{
*marco.nizama@fain.uncoma.edu.ar

†caceres@cab.cnea.gov.ar
}

states and decoherence phenomena in quantum information theory [18].

To tackle this model we derive the evolution equation for the reduced density matrix in the Fock representation. We prove that this quantum master equation (QME) is a completely positive map, that is, the infinitesimal generator fulfills the structural theorem of Kossakowsky and Lindblad [4]. Then we find its exact analytical solution. In order to see how the quantum bath will affect the quantum correlations between two indistinguishable particles, we need to define a good measure of nonclassical correlations. This is not a simple task because of symmetrization (bosons) or antisymmetrization (fermions) of the wave function (pure states) imposed by the character of indistinguishability of the particles, and so many different approaches have been proposed [19-26]. Of all possible measures of nonclassical correlations for indistinguishable particles, we follow the proposal of Wiseman and Vaccaro [19], but for mixed states [20,27]; this approach has a quite accessible numerical character. Thus, using this approach, we can choose some standard measure for the nonclassical correlation for mixed states. We use the negativity to quantify the nonclassical correlations between two indistinguishable particles [28]. Additionally, we show the total probability of finding one particle in each subsystem belonging to a chosen bipartition; in fact, in the present paper, we choose two different geometrical bipartitions. Complementary to this calculation, we calculate also the total probability of finding two particles in either subsystem and zero particles in the other (this is similar to the mirror correlation calculated for distinguishable particles [10]).

We show that the time evolution of the negativity depends strongly on the type of bipartition we have chosen. So the selection of the bipartition should be made carefully when planing an experimental setup. In fact, we show that in the presence of dissipation (a thermal bath) nonclassical correlations go to a constant value for a mirror-alternative bipartition, while for a left-right bipartition (our first defined bipartition) this constant behavior does not occur. This is an 
interesting result that has also been confirmed experimentally in fermionic systems [6-9]. In the present paper we investigate how the negativity, for indistinguishable particles, depends on the geometry we use to define a bipartition. Note that when we refer to a given geometry of a bipartition we are concerned with a set defined on a regular one-dimensional lattice.

\section{INDISTINGUISHABLE PARTICLES (MARKOV APPROACH)}

The equivalence between Fock's space and the symmetric (antisymmetric) Wannier basis for indistinguishable particles was previously established in $[10,11]$. In this section we introduce the QME for many indistinguishable particles in the second quantization approach. In the present paper the QME will be written in terms of the translation operator in Fock's representation; in this form the entanglement of the indistinguishable particles can properly be established.

The total Hamiltonian of the system $\mathcal{S}$ interacting with a common bath $\mathcal{B}$ is

$$
H_{\mathcal{T}}=H_{\mathcal{S}}+H_{\mathcal{B}}+H_{\mathcal{S B}}
$$

where $H_{\mathcal{S}}$ is the free tight-binding Hamiltonian $H_{\mathcal{S}}=$ $E_{0} \mathbf{I}-\frac{1}{2} \Omega \sum_{s=-\infty}^{\infty}\left(C_{s+1}^{\dagger} C_{s}+C_{s-1}^{\dagger} C_{s}\right)$, with $\left\{C_{s}^{\dagger}, C_{s}\right\}$ Fock's creation and annihilation operators with the lattice label $s$ and I the identity operator. In addition, $N_{p}$ and $E_{0}$ represent the number of particles and the site energy, respectively. The phonon bath Hamiltonian is defined as $H_{\mathcal{B}}=\sum_{k} \hbar \omega_{k} \mathcal{B}_{k}^{\dagger} \mathcal{B}_{k}$, where $\left\{\mathcal{B}_{k}^{\dagger}, \mathcal{B}_{k}\right\}$ are bosonic operators characterizing the thermal bath at equilibrium. In the present paper we investigate an interaction Hamiltonian which is expected in thermally activated transport processes, leading therefore to a diffusion coefficient proportional to the temperature of the bath. Thus, for the interaction between $\mathcal{S}$ and $\mathcal{B}$, we choose the Hamiltonian

$$
H_{\mathcal{S B}}=\hbar \Gamma\left(R \otimes \sum_{v} v_{v} \mathcal{B}_{v}+R^{\dagger} \otimes \sum_{v} v_{v}^{*} \mathcal{B}_{v}^{\dagger}\right)
$$

This interaction represents the action of the thermal bath to produce a translation on any indistinguishable particle. Here $v_{v}$ represents the spectral function of the phonon bath and $\Gamma$ is the interaction parameter between $\mathcal{S}$ and $\mathcal{B}$. Other interactions, for example, proportional to the position operator [29], may reproduce the electric conductivity in conducting polymers and in Fermi liquids.

Shift operators appearing in (2) acting on indistinguishable particles can be written in the form

$$
R=\sum_{s=-\infty}^{\infty} C_{s-1}^{\dagger} C_{s}, \quad R^{\dagger}=\sum_{s=-\infty}^{\infty} C_{s+1}^{\dagger} C_{s} .
$$

The two statistics (for indistinguishable particles) are taken into account by using the commutation relations

$$
\begin{aligned}
C_{s} C_{s}^{\dagger} \mp C_{s^{\prime}}^{\dagger} C_{s} & =\delta_{s, s^{\prime}}, \\
C_{s} C_{s^{\prime}} \mp C_{s^{\prime}} C_{s} & =0, \\
C_{s}^{\dagger} C_{s}^{\dagger} \mp C_{s^{\prime}}^{\dagger} C_{s}^{\dagger} & =0,
\end{aligned}
$$

where $\mp$ are used for bosons and fermions, respectively.
By introducing the Born-Markov approximation the QME can in general, for any number of particles, be written in the form

$$
\begin{aligned}
\frac{d \rho}{d t}= & \frac{-i}{\hbar}\left[H_{\mathrm{eff}}, \rho\right]+\frac{D}{2}\left(2 R \rho R^{\dagger}-R^{\dagger} R \rho-\rho R R^{\dagger}\right) \\
& +\frac{D}{2}\left(2 R^{\dagger} \rho R-R R^{\dagger} \rho-\rho R R^{\dagger}\right)
\end{aligned}
$$

(for more details see Appendix). Here the dissipative parameter is $D=\Gamma^{2} k_{B} T / \hbar$, with $T$ representing the temperature of the bath $\mathcal{B}$. Having that $E_{0}$ is the site energy and using that it is constant for all sites, without loss of generality, we can set $N_{p} E_{0}=\Omega$, wherewith the effective Hamiltonian can be written as

$$
H_{\mathrm{eff}}=\Omega\left(\mathbf{I}-\frac{R^{\dagger}+R}{2}\right)-\hbar \omega_{c} R R^{\dagger},
$$

where $\omega_{c}$ is proportional to the frequency cutoff in the Ohmic approximation $[12,13]$. In Refs. $[10,11]$ it was noted that the terms $R^{\dagger} R \rho$ and $R R^{\dagger} \rho$ generate coherence, while $R^{\dagger} \rho R$ and $R \rho R^{\dagger}$ are responsible for inducing decoherence in the system.

\section{SOLUTION OF THE QME (TWO PARTICLES)}

To solve the QME (4) and to find an analytical solution, we use the Fourier representation because both the von Neumann and the Kossakowsky-Lindblad (KL) superoperators can be diagonalized on this basis. In order to simplify the analysis of the QME (4) we will omit the term for the frequency cutoff $\hbar \omega_{c} R R^{\dagger}$ in the effective Hamiltonian. This term only produces additional reversible coherence [10].

\section{A. Fourier analytical solution}

The Fourier representation is characterized by operators in the second quantization

$$
C_{k}^{\dagger}=\frac{1}{\sqrt{2 \pi}} \sum_{s=-\infty}^{\infty} e^{i k s} C_{s}^{\dagger}, \quad C_{k}=\frac{1}{\sqrt{2 \pi}} \sum_{s=-\infty}^{\infty} e^{-i k s} C_{s} .
$$

In the present paper we are interested in studying the dynamics of two indistinguishable particles (the number of particles is conserved for the present Hamiltonian model). We will characterize the system $\mathcal{S}$ by the two-particle state (in Fourier's representation)

$$
\left|k_{1}, k_{2}\right\rangle=C_{k_{1}}^{\dagger} C_{k_{2}}^{\dagger}|\phi\rangle
$$

where $|\phi\rangle$ is the vacuum state. Using that

$$
\begin{aligned}
& R^{\dagger} C_{s_{1}}^{\dagger} C_{s_{2}}^{\dagger}|\phi\rangle=C_{s_{1}+1}^{\dagger} C_{s_{2}}^{\dagger}|\phi\rangle+C_{s_{1}}^{\dagger} C_{s_{2}+1}^{\dagger}|\phi\rangle, \\
& R C_{s_{1}}^{\dagger} C_{s_{2}}^{\dagger}|\phi\rangle=C_{s_{1}-1}^{\dagger} C_{s_{2}}^{\dagger}|\phi\rangle+C_{s_{1}}^{\dagger} C_{s_{2}-1}^{\dagger}|\phi\rangle,
\end{aligned}
$$

as well as (5) in the QME (4), the analytical solution in the Fourier representation can be found after some algebra. The QME can be written as

$$
\frac{d}{d t}\left\langle k_{1}, k_{2}|\rho(t)| k_{1}^{\prime}, k_{2}^{\prime}\right\rangle=\mathcal{F}\left(k_{1}, k_{1}^{\prime}, k_{2}, k_{2}^{\prime}\right)\left\langle k_{1}, k_{2}|\rho(t)| k_{1}^{\prime}, k_{2}^{\prime}\right\rangle,
$$


where $\mathcal{F}\left(k_{1}, k_{1}^{\prime}, k_{2}, k_{2}^{\prime}\right)$ is the KL infinitesimal generator [4]. Then the solution of the QME in the Fourier basis is

$$
\left\langle k_{1}, k_{2}|\rho(t)| k_{1}^{\prime}, k_{2}^{\prime}\right\rangle=e^{\mathcal{F}\left(k_{1}, k_{1}^{\prime}, k_{2}, k_{2}^{\prime}\right) t}\left\langle k_{1}, k_{2}|\rho(0)| k_{1}^{\prime}, k_{2}^{\prime}\right\rangle,
$$

with

$$
\begin{aligned}
\mathcal{F}\left(k_{1}, k_{1}^{\prime}, k_{2}, k_{2}^{\prime}\right) \equiv & {\left[\mathcal{F}^{(1)}\left(k_{1}, k_{1}^{\prime}\right)+\mathcal{F}^{(1)}\left(k_{2}, k_{2}^{\prime}\right)\right] } \\
& +2 D\left[\mathbf{C}\left(k_{1}, k_{2}^{\prime}\right)+\mathbf{C}\left(k_{2}, k_{1}^{\prime}\right)\right. \\
& \left.-\mathbf{C}\left(k_{1}, k_{2}\right)-\mathbf{C}\left(k_{1}^{\prime}, k_{2}^{\prime}\right)\right],
\end{aligned}
$$

where

$$
\mathcal{F}^{(1)}\left(k_{i}, k_{i}^{\prime}\right) \equiv\left[\frac{-i}{\hbar}\left(\mathcal{E}_{k_{i}}-\mathcal{E}_{k_{i}^{\prime}}\right)+2 D\left(\mathbf{C}\left(k_{i}, k_{i}^{\prime}\right)-1\right)\right]
$$

is the one-particle infinitesimal generator in the Fourier representation, with

$$
\mathcal{E}_{k_{i}} \equiv \Omega\left(1-\cos k_{i}\right)
$$

that is, the eigenenergy of one free particle in the lattice [13]. In (10) we have defined the function

$$
\mathbf{C}\left(k_{1}, k_{2}\right) \equiv \cos \left(k_{1}-k_{2}\right),
$$

which produces bath-induced correlation between particles.

\section{B. Analytical solution in the lattice}

The solution (9) is similar to the case for distinguishable particles (see $[10,11]$ ); the difference is in the use of Fock's vector states leading to its indistinguishable character. Noting that

$$
C_{s}^{\dagger}=\frac{1}{\sqrt{2 \pi}} \int_{-\pi}^{\pi} e^{-i k s} C_{k}^{\dagger} d k, \quad C_{s}=\frac{1}{\sqrt{2 \pi}} \int_{-\pi}^{\pi} e^{+i k s} C_{k} d k,
$$

in Fock's lattice mode the solution is

$$
\left\langle s_{1}, s_{2}|\rho(t)| s_{1}^{\prime}, s_{2}^{\prime}\right\rangle=\left\langle\phi\left|C_{s_{1}} C_{s_{2}} \rho(t) C_{s_{1}^{\prime}}^{\dagger} C_{s_{2}^{\prime}}^{\dagger}\right| \phi\right\rangle,
$$

which can be written, returning to the lattice representation, as

$$
\begin{aligned}
\left\langle s_{1}, s_{2}|\rho(t)| s_{1}^{\prime}, s_{2}^{\prime}\right\rangle= & \frac{1}{(2 \pi)^{2}} \int_{-\pi}^{\pi} \int_{-\pi}^{\pi} \int_{-\pi}^{\pi} \int_{-\pi}^{\pi} d k_{1} d k_{1}^{\prime} d k_{2} d k_{2}^{\prime} \\
& \times\left\langle k_{1}, k_{2}|\rho(0)| k_{1}^{\prime}, k_{2}^{\prime}\right\rangle \\
& \times e^{i k_{1} s_{1}} e^{-i k_{1}^{\prime} s_{1}^{\prime}} e^{i k_{2} s_{2}} e^{-i k_{2}^{\prime} s_{2}^{\prime}} e^{\mathcal{F}\left(k_{1}, k_{1}^{\prime}, k_{2}, k_{2}^{\prime}\right) t},
\end{aligned}
$$

where

$$
\left|s_{1}, s_{2}\right\rangle \equiv\left|\ldots, 0,1_{s_{1}}, 0, \ldots, 0,1_{s_{2}}, 0, \ldots\right\rangle .
$$

This is the mode-occupation basis, which represents two particles at sites $s_{1}$ and $s_{2}$ on the lattice.

A general initial condition (IC) in the lattice will be

$$
|\Psi(0)\rangle_{l_{1}, l_{2}}=\alpha\left(l_{1}, l_{2}\right) C_{l_{1}}^{\dagger} C_{l_{2}}^{\dagger}|\phi\rangle .
$$

Therefore, the IC $\rho(0)$, in (15), can be written as

$$
\rho(0)=|\Psi(0)\rangle_{l_{1}^{\prime}, l_{2}^{\prime}}\left\langle\left.\Psi(0)\right|_{l_{1}, l_{2}} .\right.
$$

Thus the IC gets the form

$$
\begin{aligned}
\left\langle k_{1}, k_{2}|\rho(0)| k_{1}^{\prime}, k_{2}^{\prime}\right\rangle= & \left\{e^{-i k_{1} l_{1}} e^{-i k_{2} l_{2}} e^{+i k_{1}^{\prime} l_{1}^{\prime}} e^{+i k_{2}^{\prime} l_{2}^{\prime}}\right. \\
& -\beta e^{-i k_{1} l_{1}} e^{-i k_{2} l_{2}} e^{+i k_{1}^{\prime} l_{2}^{\prime}} e^{+i k_{2}^{\prime} l_{1}^{\prime}} \\
& -\beta e^{-i k_{1} l_{2}} e^{-i k_{2} l_{1}} e^{+i k_{1}^{\prime} l_{1}^{\prime}} e^{+i k_{2}^{\prime} l_{2}^{\prime}} \\
& \left.+e^{-i k_{1} l_{2}} e^{-i k_{2} l_{1}} e^{+i k_{1}^{\prime} l_{2}^{\prime}} e^{+i k_{2}^{\prime} l_{1}^{\prime}}\right\} \frac{A}{(2 \pi)^{2}},
\end{aligned}
$$

where

$$
A=\alpha\left(l_{1}, l_{2}\right) \alpha\left(l_{1}^{\prime}, l_{2}^{\prime}\right)^{*}, \quad \beta= \begin{cases}1 & \text { (for bosons) } \\ -1 & \text { (for fermions) }\end{cases}
$$

and so

$$
\left\langle\phi\left|C_{n_{1}} C_{n_{2}} C_{r_{1}}^{\dagger} C_{r_{2}}^{\dagger}\right| \phi\right\rangle=\delta_{n_{1}, r_{2}} \delta_{n_{2}, r_{1}}-\beta \delta_{n_{2}, r_{2}} \delta_{n_{1}, r_{1}} .
$$

In order to analyze quantum correlations, we are interested in studying lattice bipartitions, so we introduce initial conditions with the index $l_{1}=-l_{2}=p$, that is, with $\alpha(p,-p)=$ $1 / \sqrt{2}$. For example,

$$
|\Psi(0)\rangle_{p,-p}=\frac{1}{\sqrt{2}} C_{-p}^{\dagger} C_{p}^{\dagger}|\phi\rangle
$$

and

$$
|\Psi(0)\rangle=\frac{1}{\sqrt{2}} \sum_{j=1}^{2} C_{-p_{j}}^{\dagger} C_{p_{j}}^{\dagger}|\phi\rangle .
$$

Thus, in the lattice the IC of $\rho$ gets the form

$$
\begin{aligned}
\rho(0)= & \frac{1}{2} C_{-p_{1}}^{\dagger} C_{p_{1}}^{\dagger}|\phi\rangle\left\langle\phi\left|C_{p_{1}} C_{-p_{1}}+\frac{1}{2} C_{-p_{1}}^{\dagger} C_{p_{1}}^{\dagger}\right| \phi\right\rangle\langle\phi| C_{p_{2}} C_{-p_{2}} \\
& +\frac{1}{2} C_{-p_{2}}^{\dagger} C_{p_{2}}^{\dagger}|\phi\rangle\left\langle\phi\left|C_{p_{1}} C_{-p_{1}}+\frac{1}{2} C_{-p_{2}}^{\dagger} C_{p_{2}}^{\dagger}\right| \phi\right\rangle\langle\phi| C_{p_{2}} C_{-p_{2}} .
\end{aligned}
$$

We will use, for example, $p_{1}=1$ and $p_{2}=2$. Then it is possible to see that the solution of the QME for bosons or fermions has an analytical form. To do this task we apply some general properties of Bessel functions [30] and we define several parameters in order to simplify its expression, that is, $t_{\Omega} \equiv \frac{\Omega t}{\hbar}$ and $t_{D} \equiv 2 D t$. After some algebra it is possible to check that $\rho(t)$ is Hermitian, positive definite, and satisfies the normalization condition.

\section{DIRECT AND INDIRECT INDICATORS OF CORRELATIONS}

\section{A. Negativity of indistinguishable particles}

In general, measuring entanglement for indistinguishable particles is not a simple task (because of the exchange symmetry of the quantum state); this has led to many extensive studies [19-26]. One such measurement was proposed by Wiseman and Vacaro [19], who offered a simple operational procedure.

The entanglement $E_{p}$ for two particles can be calculated as

$$
E_{p}=P_{1,1} \varepsilon\left(\rho_{1,1}\right),
$$

where $\rho_{1,1} \equiv \prod_{1,1} \rho_{A B} \prod_{1,1}$ is the projected state of $\rho_{A B}$ and $\prod_{1,1}$ represents the projection onto one vector state with one particle in each subsystem (of course a given bipartition is necessary to define this measure) $[19,20,27]$. For example, in 


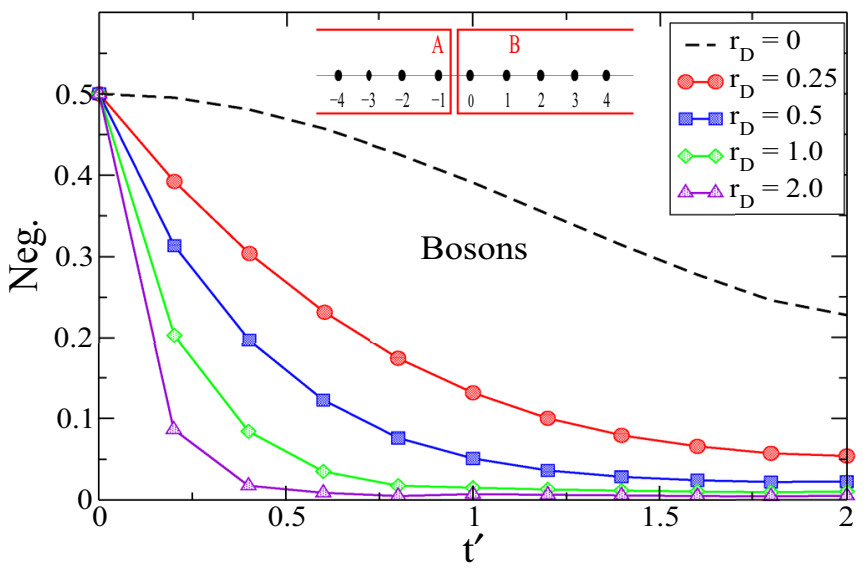

FIG. 1. Quantum correlation for two bosons (negativity) as a function of the dimensionless time $t^{\prime}$ for several values of the dissipative parameter $r_{D}=0,0.25,0.5,1.0,2.0$. The inset shows the bipartition used in (20).

Ref. [27] Beggi et al. proposed the measurement of quantum discord based on the definition of entanglement of particles given by Wiseman and Vaccaro. The probability of finding one particle in each subsystem is denoted by $P_{1,1}$, and $\varepsilon\left(\rho_{1,1}\right)$ represents a bipartite entanglement measure which estimates the quantity of nonclassical correlations.

There are different measures for characterizing quantum correlations of mixed quantum state (see, e.g., Refs. [31,32]). One of them is the negativity proposed by Vidal and Werner [28]. This measure is straightforward to calculate for the bipartite mixed state $\rho_{A B} \equiv \rho$. The reduced partial transpose of the density matrix in the space $A$ or $B$ is required. In this context the matrix elements of the partial transpose of $\rho$ are defined as (in space $A$ )

$$
\left\langle i_{A}, j_{B}\left|\rho^{T_{A}}\right| i_{A}^{\prime}, j_{B}^{\prime}\right\rangle \equiv\left\langle i_{A}^{\prime}, j_{B}|\rho| i_{A}, j_{B}^{\prime}\right\rangle,
$$

where the orthonormal product basis is defined as $\left|i_{A}, j_{B}\right\rangle \equiv$ $\left|i_{A}\right\rangle \otimes\left|j_{B}\right\rangle \in \mathcal{H}_{A} \otimes \mathcal{H}_{B}$, with $\mathcal{H}_{A(B)}$ representing the Hilbert space in $A(B)$ [in the present case the basis is in the Fock representation (16)]. Using (21), the negativity is calculated as

$$
N(\rho)=\frac{\left\|\rho^{T_{A}}\right\|-1}{2},
$$

where $\|\cdot\|$ represents the trace norm [28]. From (22), the negativity can also be expressed as

$$
N(\rho)=\sum_{i}\left|\mu_{i}\right|
$$

where $\mu_{i}$ is a negative eigenvalue of $\rho^{T_{A}}$. Therefore, it is straightforward to obtain the negativity for a bipartite mixed state.

\section{Left-right bipartition}

In order to study quantum correlations between the particles, first we define the left-right bipartition $\{A, B\}$ such that subsystem $A$ has the negative sites on the lattice and $B$ the non-negative sites (the left-right bipartition is shown in the inset of Fig. 1). In Fig. 1 we show the quantum

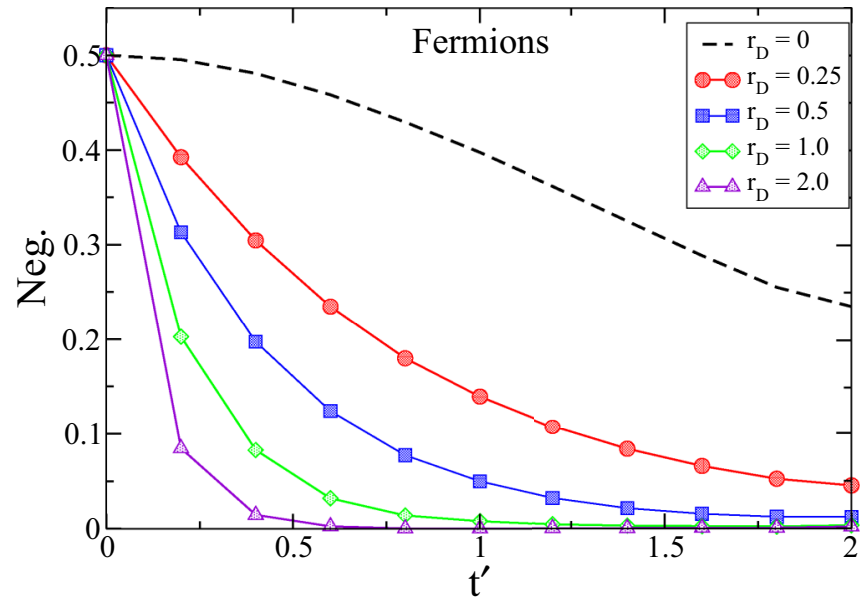

FIG. 2. Quantum correlation for two fermions (negativity) as function of dimensionless time $t^{\prime}$ and the same values of $r_{D}$ and bipartition as in Fig. 1. It decreases with increasing $t^{\prime}$ or $r_{D}$.

correlation (negativity) between two bosons for a correlated initial condition (19) with sites $p_{1}=1$ and $p_{2}=2$. Here we define a dissipative parameter as $r_{D}=\Omega \hbar / 2 D$ and use the dimensionless time $t^{\prime}=t_{\Omega} \equiv \Omega t / \hbar$ for convenience. We note that negativity decreases with increasing time and/or dissipation; even for $r_{D}=0$ the negativity is reduced for $t^{\prime}>0$. The latter is due to the fact that the chosen bipartition does not consider quantum correlations between correlated internal states in $A$ or $B$, for instance, correlations between sites -1 and -2 (or 1 and 2). Figure 1 shows how correlations between particles are reduced by the presence of dissipation.

In the same way, for fermions the negativity decreases with increasing time and/or dissipation, as can be seen in Fig. 2. There is not a big difference in the behavior of the negativity for bosons and fermions, as was also pointed out in [19]. The small variations that we can see here are due to the Pauli exclusion principle (double occupation at sites on the lattice in the subsystems $A$ or $B$ are not allowed).

\section{Mirror-alternative bipartition}

Now we define another bipartition $\{A, B\}$, each having alternative sites on the whole lattice (the set $B$ includes the origin; see the inset in Fig. 3). Using this (second) bipartition, the geometry of the set allows us to detect the overlap in neighboring sites.

This set $\{A, B\}$ helps one understand how quantum correlations between the particles depend on the chosen bipartition. In this case and for $r_{D}=0$, the negativity for bosons and fermions has an oscillatory behavior in time because the mirror-alternative bipartition considers correlations between neighboring sites throughout the entire lattice (see Figs. 3 and 4). For $r_{D}>0$ (in the presence of dissipation) quantum correlations for the mirror-alternative bipartition are slightly larger than for the first bipartition. We can conclude that it is important to choose a good bipartition, on the lattice, in order to obtain a higher nonclassical correlation for $r_{D}>0$. 


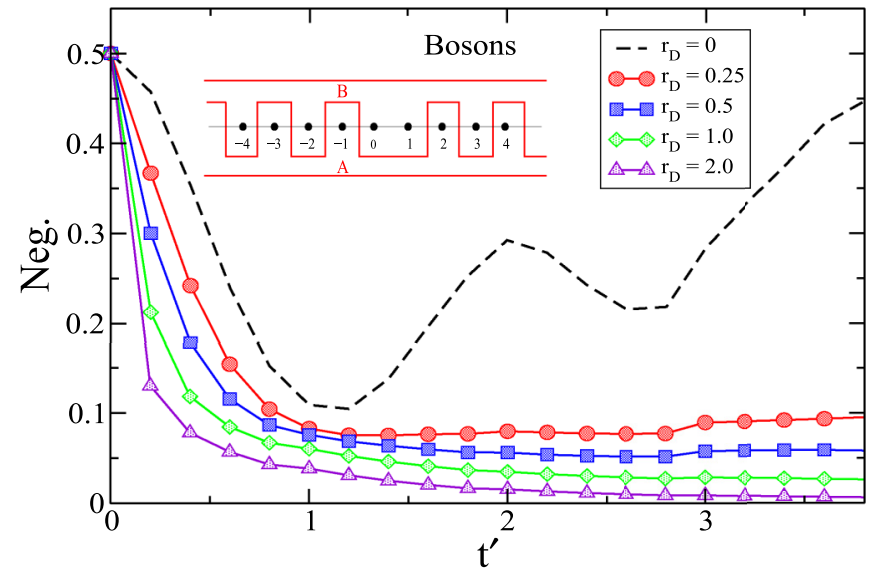

FIG. 3. Negativity for two bosons as a function of the dimensionless time $t^{\prime}$ for the same values of the dissipative parameter $r_{D}$ as in Fig. 1. The studied bipartition is represented in the inset.

\section{B. Quantum probability of finding the particles}

Another possible indirect measure of the instantaneous correlation between particles can be given in terms of the total probability function of finding one particle $P_{1,1}$ in each subsystem $A$ and $B$ (see the bipartitions studied above). This function is defined from the two-particle density matrix evaluated as

$$
P_{1,1}=\sum_{s_{A}} \sum_{s_{B}}\left\langle s_{A}, s_{B}|\rho(t)| s_{A}, s_{B}\right\rangle .
$$

Another complementary measure is the total probability function of finding two particles in $A$ (none in $B$ ), $P_{2,0}$, or zero particles in $A$ (two particles in $B$ ), $P_{0,2}$,

$$
\begin{aligned}
& P_{2,0}=\sum_{s_{A}, s_{A^{\prime}}}\left\langle s_{A}, s_{A^{\prime}}|\rho(t)| s_{A}, s_{A^{\prime}}\right\rangle, \\
& P_{0,2}=\sum_{s_{B}, s_{B^{\prime}}}\left\langle s_{B}, s_{B^{\prime}}|\rho(t)| s_{B}, s_{B^{\prime}}\right\rangle,
\end{aligned}
$$

where $\left|s_{R_{1}}, s_{R_{2}}\right\rangle$ is given by (16) (in the Fock representation), with $R_{1}, R_{2} \in A, B$ (according to the case).

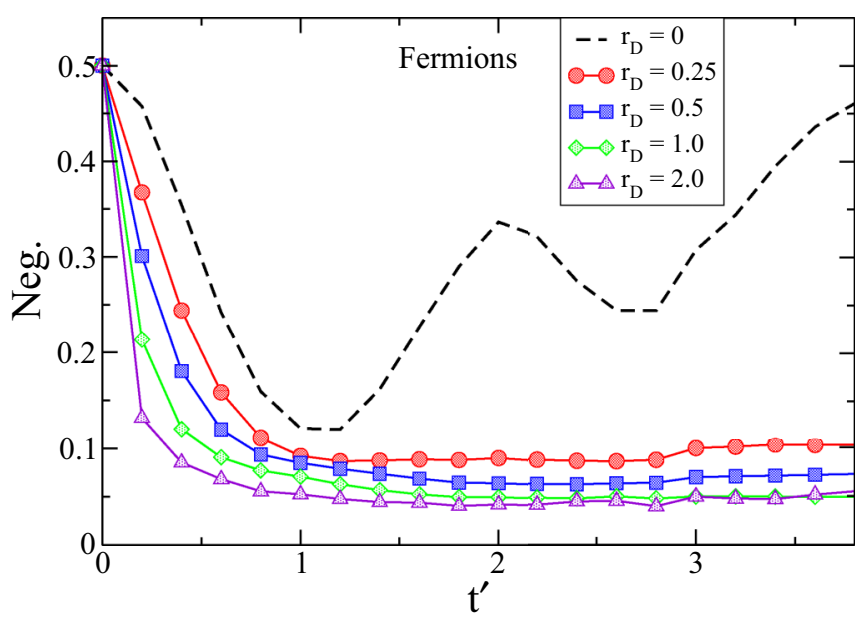

FIG. 4. Negativity of indistinguishable particles (two fermions) as function of dimensionless time $t^{\prime}$ and the same values of $r_{D}$ and bipartition as in Fig. 3.

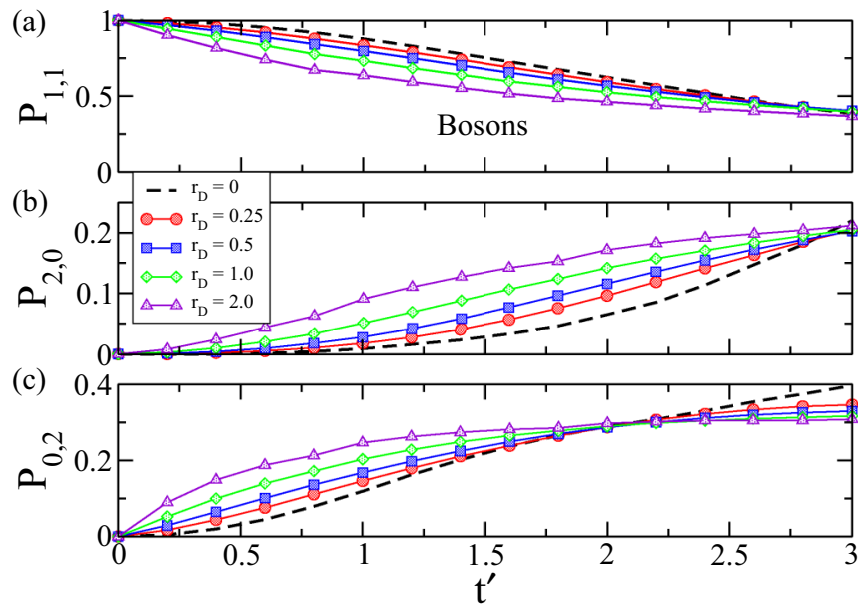

FIG. 5. (a) Probability of finding one particle $P_{1,1}$ in $A$ and $B$ as a function of dimensionless time $t^{\prime}$ and several values of $r_{D}$. (b) Probability of finding two particles in $A$. (c) Probability of finding two particles in $B$. The bipartition is at left and right sites with respect to the origin of the lattice.

\section{Left-right bipartition}

The total probability of finding the particles in either subsystem is given by (24)-(26) for this particular bipartition (see the inset in Fig. 1), that is, $s_{A}=\{-\infty, \ldots,-3,-2,-1\}$ and $s_{B}=\{0,1,2,3, \ldots, \infty\}$ (representing sites in subsystems $A$ and $B$, respectively). In Fig. 5 we have plotted these functions for bosons $P_{1,1}$ (total probability of finding one particle in each subsystem) and $P_{2,0}$ and $P_{0,2}$ (the total probability of finding two particles in one subsystem) as a function of the dimensionless time $t^{\prime}=t_{\Omega}$ and for several values of the dissipative parameter $r_{D}=\Omega \hbar / 2 D$. The IC has one particle in each subsystem [the IC is given by (19)]; then, for $t^{\prime}>0$ and/or $r_{D}>0$, the probability $P_{1,1}$ decreases; $P_{2,0}$ and $P_{0,2}$ show a complementary behavior. The fact that $P_{1,1}>0$ for $r_{D}$ and $t^{\prime}$ different from zero means that quantum correlations between particles is not null. Nevertheless, quantum correlations are destroyed by the presence of the thermal bath. On the other hand, the small differences between $P_{2,0}$ and $P_{0,2}$ are due to the fact that the bipartition is not symmetric (subsystem $B$ has the origin of the lattice). The quantum correlation between fermions is very similar to the bosonic case and for that reason we do not show the results. We noted that similar quantum correlations have been reported by Wiseman and Vaccaro [19].

\section{Mirror-alternative bipartition}

For completeness, we calculate $P_{1,1}, P_{2,0}$, and $P_{0,2}$ in the second bipartition (see the graphical representation in the inset in Fig. 3) with $s_{A}=\{-\infty, \cdots,-3,-1,2,4, \cdots, \infty\}$ and $s_{B}=\{-\infty, \cdots,-4,-2,0,1,3, \cdots, \infty\}$. In Fig. 6 we show the results of the probability for this bipartition as a function of $t^{\prime}$ and $r_{D}$ (for two bosons). The case for fermions is very similar (see the results for the left-right bipartition). In contrast with the first bipartition, the functions $P_{1,1}, P_{2,0}$, and $P_{0,2}$ have an oscillatory behavior. For $t^{\prime}=0, P_{1,1}$ has its maximum value, which decreases with increasing $t^{\prime}$ and $r_{D}$ and seems to converge to $1 / 2 ; P_{2,0}$ and $P_{0,2}$ show a 


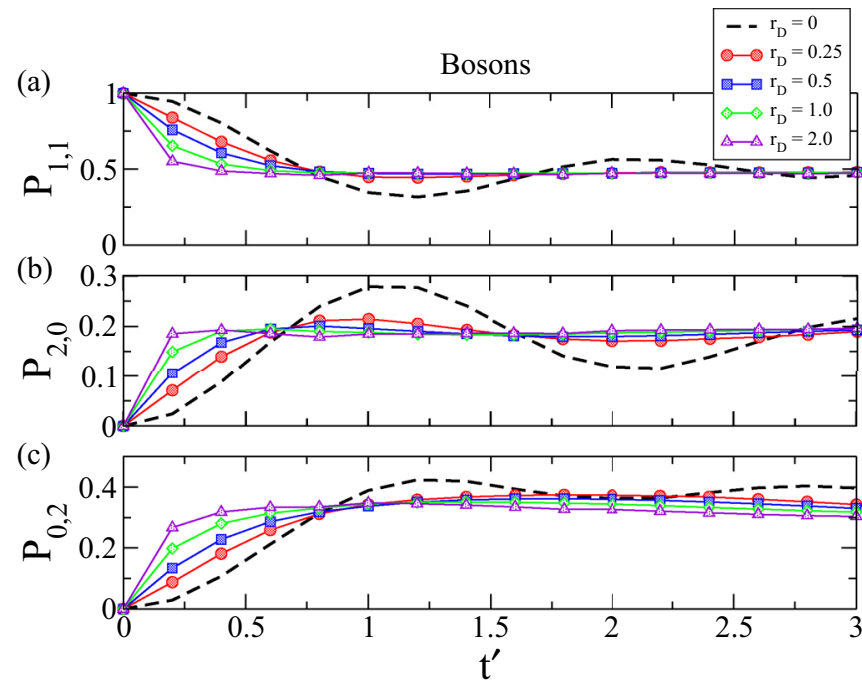

FIG. 6. Probability (a) $P_{1,1}$, (b) $P_{2,0}$, and (c) $P_{0,2}$ as a function of dimensionless time $t^{\prime}$ and $r_{D}=0,0.25,0.5,1.0,2.0$ for the mirroralternative bipartition (see the inset in Fig. 3).

complementary behavior to $P_{1,1}$. In this case, $P_{2,0}$ and $P_{0,2}$ are not equal, because the mirror-alternative bipartition is not symmetric with respect to an exchange of $A$ and $B$. Therefore, we can say that the mirror quantum probability supports and explains our previous results obtained with the negativity measure (Sec. IV A).

\section{CONCLUSION}

We have worked out, in a one-dimensional lattice, two indistinguishable particles coupled to a common phonon bath $\mathcal{B}$. We have solved the density matrix in the Markov approximation and an analytical solution for bosons and fermions has been found in the Fock representation.

Quantum correlations between two indistinguishable particles (bosons or fermions) have been studied in the presence of a thermal quantum bath. These correlations depend on the chosen bipartition. However, small differences are found between them because of the Pauli exclusion principle.

In general, the bath destroys correlations but for fermions correlations seem to saturate to a constant value for a finite value of the dissipative parameter $r_{D}$, in the mirror-alternative bipartition case.

The analysis of the negativity has been supported by the study of the total probability of finding one, two, or no particles in each subsystem $\{A, B\}$.

Different coupling interaction between $\mathcal{S}$ and $\mathcal{B}$ [Hamiltonian (2)] may have important implications in the analysis of the efficiency to obtain greater values of quantum correlations.

\section{ACKNOWLEDGMENT}

M.O.C and M.N. gratefully acknowledge support received from CONICET, Argentina, through Grant No. PIP 112201501-00216 CO.

\section{APPENDIX: THE THERMALLY ACTIVATED QME FOR FREE INDISTINGUISHABLE PARTICLES}

Here we derive in a Fock representation the QME for free indistinguishable particles interacting with a thermal bosonic bath. A similar demonstration has been done for a single Levy-like free particle in a lattice [12].

The total Hamiltonian of the system $\mathcal{S}$ interacting with a common bath $\mathcal{B}$ is

$$
\begin{aligned}
H_{\mathcal{T}}= & E_{0} \mathbf{I}-\frac{\Omega}{2} \sum_{s=-\infty}^{\infty}\left(C_{s+1}^{\dagger} C_{s}+C_{s-1}^{\dagger} C_{s}\right) \\
& +\sum_{k} \hbar \omega_{k} \mathcal{B}_{k}^{\dagger} \mathcal{B}_{k}+H_{\mathcal{S B}},
\end{aligned}
$$

where $\left\{C_{s}^{\dagger}, C_{s}\right\}$ are Fock's creation and annihilation operators with the lattice label $s$ and $\mathbf{I}$ is the identity operator. In addition, $N_{p}$ and $E_{0}$ represent the number of particles and the site energy, respectively. The bath Hamiltonian is defined in terms of bosonic operators $\left\{\mathcal{B}_{k}^{\dagger}, \mathcal{B}_{k}\right\}$ characterizing the thermal bath at equilibrium. In the present paper we investigate an interaction Hamiltonian $H_{\mathcal{S B}}$ given by

$$
H_{\mathcal{S B}}=\hbar \Gamma\left(R \otimes \sum_{\nu} v_{\nu} \mathcal{B}_{v}+R^{\dagger} \otimes \sum_{\nu} v_{v}^{*} \mathcal{B}_{v}^{\dagger}\right),
$$

where the system's operators $R$ and $R^{\dagger}$ are written in terms of the Fock operators

$$
R=\sum_{s=-\infty}^{\infty} C_{s-1}^{\dagger} C_{s}, \quad R^{\dagger}=\sum_{s=-\infty}^{\infty} C_{s+1}^{\dagger} C_{s} .
$$

The interaction (A2) represents the action of the thermal bath $\mathcal{B}$ to produce a translation on any indistinguishable particle of system $\mathcal{S}$. Here $v_{v}$ gives the spectral function characterizing the phonon bath and $\Gamma$ is the interaction parameter. As we mentioned before, $\left\{C_{s_{1}}^{\dagger}, C_{s_{2}}^{\dagger}\right\}$ are Fock's creation operators; both operators acting on the vacuum state $|\phi\rangle$ will produce a two-particle vector state

$$
C_{s_{1}}^{\dagger} C_{s_{2}}^{\dagger}|\phi\rangle=\left|\ldots, 0,1_{s_{1}}, 0, \ldots, 0,1_{s_{2}}, 0, \ldots\right\rangle,
$$

which has been created by two indistinguishable particles at sites $s_{1}$ and $s_{2}$. If these particles are bosonic, the operators $C_{s_{1}}^{\dagger}, C_{s_{2}}^{\dagger}$ and $C_{S_{1}}, C_{s_{2}}$ satisfy the usual relations

$$
\left[C_{s_{1}}, C_{s_{2}}^{\dagger}\right]=\delta_{s_{1}, s_{2}}, \quad\left[C_{s_{1}}, C_{s_{2}}\right]=\left[C_{s_{1}}^{\dagger}, C_{s_{2}}^{\dagger}\right]=0 .
$$

In (A2) we can recognize that $R$ and $R^{\dagger}$ are translation operators in the one-dimensional lattice; for example, using (A5) we can prove that $R$ translates back (individually) two indistinguishable particles

$$
\begin{aligned}
R C_{s_{1}}^{\dagger} C_{s_{2}}^{\dagger}|\phi\rangle & =\sum_{s=-\infty}^{\infty} C_{s-1}^{\dagger} C_{s} C_{s_{1}}^{\dagger} C_{s_{2}}^{\dagger}|\phi\rangle \\
& =\sum_{s=-\infty}^{\infty} C_{s-1}^{\dagger}\left(\delta_{s, s_{1}}+C_{s_{1}}^{\dagger} C_{s}\right) C_{s_{2}}^{\dagger}|\phi\rangle \\
& =C_{s_{1}-1}^{\dagger} C_{s_{2}}^{\dagger}|\phi\rangle+\sum_{s=-\infty}^{\infty} C_{s-1}^{\dagger} C_{s_{1}}^{\dagger}\left(\delta_{s, s_{2}}+C_{s_{2}}^{\dagger} C_{s}\right)|\phi\rangle \\
& =C_{s_{1}-1}^{\dagger} C_{s_{2}}^{\dagger}|\phi\rangle+C_{s_{2}-1}^{\dagger} C_{s_{1}}^{\dagger}|\phi\rangle,
\end{aligned}
$$


where we have used that $C_{s}|\phi\rangle=0$. In a similar way, noting that

$$
R^{\dagger}=\sum_{s=-\infty}^{\infty}\left(C_{s-1}^{\dagger} C_{s}\right)^{\dagger}=\sum_{s=-\infty}^{\infty} C_{s}^{\dagger} C_{s-1}=\sum_{s^{\prime}=-\infty}^{\infty} C_{s^{\prime}+1}^{\dagger} C_{s^{\prime}},
$$

it is simple to prove that $R^{\dagger}$ translates forward:

$$
R^{\dagger} C_{s_{1}}^{\dagger} C_{s_{2}}^{\dagger}|\phi\rangle=C_{s_{1}+1}^{\dagger} C_{s_{2}}^{\dagger}|\phi\rangle+C_{s_{2}+1}^{\dagger} C_{s_{2}}^{\dagger}|\phi\rangle
$$

To calculate the infinitesimal Kossakoswki-Lindbland generator it is necessary to know the action of the operator $R^{\dagger} R$ on an arbitrary Fock vector state. To calculate this operator, we use the definitions of $R^{\dagger}, R$, and (A5) to obtain

$$
\begin{aligned}
R R^{\dagger} & =\sum_{s=-\infty}^{\infty} C_{s-1}^{\dagger} C_{s} \sum_{s^{\prime}=-\infty}^{\infty} C_{s^{\prime}+1}^{\dagger} C_{s^{\prime}} \\
& =\sum_{s^{\prime}=-\infty}^{\infty} C_{s^{\prime}}^{\dagger} C_{s^{\prime}}+\sum_{s=-\infty}^{\infty} \sum_{s^{\prime}=-\infty}^{\infty} C_{s-1}^{\dagger} C_{s^{\prime}+1}^{\dagger} C_{s} C_{s^{\prime}}
\end{aligned}
$$

Therefore, for example, applying $R R^{\dagger}$ to a two-particle vector state, we get

$$
\begin{aligned}
R R^{\dagger} C_{s_{1}}^{\dagger} C_{s_{2}}^{\dagger}|\phi\rangle= & 2 C_{s_{1}}^{\dagger} C_{s_{2}}^{\dagger}|\phi\rangle+C_{s_{1}-1}^{\dagger} C_{s_{2}+1}^{\dagger}|\phi\rangle \\
& +C_{s_{1}+1}^{\dagger} C_{s_{2}-1}^{\dagger}|\phi\rangle .
\end{aligned}
$$

In a similar way we can also prove that $R R^{\dagger}=R^{\dagger} R$ and therefore $R$ and $R^{\dagger}$ commute, i.e., $\left[R, R^{\dagger}\right]=0$. These are important relations necessary to calculate the QME when eliminating the bath degree of freedoms. We remark that a similar calculation can be done for fermionic particles.

The QME can be obtained by eliminating the variables of the thermal bath $\mathcal{B}$ and assuming for the initial condition of the total density matrix a direct product $\rho_{T}(0)=$ $\rho(0) \otimes \rho_{\mathcal{B}}^{e q}$, where $\rho_{\mathcal{B}}^{e q}$ is the equilibrium density matrix of $\mathcal{B}$. Then introducing a second-order perturbation (Born-Markov approximation), we can arrive at an infinitesimal generator [3]

$$
\dot{\rho}=\frac{-i}{\hbar}\left[H_{\mathrm{eff}}, \rho\right]+\int_{0}^{\infty} \operatorname{Tr}_{\mathcal{B}}\left\{\left[H_{\mathcal{S B}},\left[H_{\mathcal{S B}}(-\tau), \rho_{\mathcal{B}}^{e q} \rho(t)\right]\right]\right\} d \tau .
$$

The crucial point is to ensure that this infinitesimal generator has a KL structure (a completely positive condition); this fact strongly depends on the interaction model. For an interaction Hamiltonian of the form (A2), that is, $H_{\mathcal{S B}}=$ $\sum_{\beta=1}^{2} V_{\beta} \otimes \mathcal{W}_{\beta}$, the QME can be written as

$$
\dot{\rho}=\frac{-i}{\hbar}\left[H_{\mathrm{eff}}, \rho\right]+F[\rho]-\frac{1}{2}\left\{F^{*}[\mathbf{1}], \rho\right\}_{+} .
$$

Here the operator $\left\{F^{*}[\mathbf{1}], \bullet\right\}$ can be regarded as a dissipative operator and $F[\bullet]$ as a fluctuating one. The effective Hamiltonian is given by

$$
\begin{aligned}
H_{\mathrm{eff}}= & H_{\mathcal{S}}-\frac{i}{2 \hbar} \sum_{\alpha, \beta=1}^{2} \int_{0}^{\infty} d \tau\left[\chi_{\alpha \beta}(-\tau) V_{\alpha}^{\dagger} V_{\beta}(-\tau)\right. \\
& \left.-\chi_{\alpha \beta}^{*}(-\tau) V_{\beta}^{\dagger}(-\tau) V_{\alpha}\right]
\end{aligned}
$$

where $\chi_{\alpha \beta}(-\tau) \equiv \operatorname{Tr}_{\mathcal{B}}\left[\rho_{\mathcal{B}}^{e q} \mathcal{W}_{\alpha}^{\dagger} \mathcal{W}_{\beta}(-\tau)\right]$ are bath thermal correlations. The superoperator $F[\bullet]$ (and its dual $F^{*}[\bullet]$ ) appearing in (A11) is given by

$$
\begin{aligned}
F[\bullet]= & \frac{1}{\hbar^{2}} \sum_{\alpha, \beta=1}^{2} \int_{0}^{\infty} d \tau\left[\chi_{\alpha \beta}(-\tau) V_{\beta}(-\tau) \bullet V_{\alpha}^{\dagger}\right. \\
& \left.+\chi_{\alpha \beta}^{*}(-\tau) V_{\alpha} \bullet V_{\beta}^{\dagger}(-\tau)\right] .
\end{aligned}
$$

In our case $V_{1}=V_{2}^{\dagger}=\hbar \Gamma R$, with $\Gamma>0$, and $\mathcal{W}_{1}=\mathcal{W}_{2}^{\dagger}=$ $\sum_{v} v_{v} \mathcal{B}_{\nu}$, and so the $\mathrm{KL}$ structure is ensured because $\left[R, H_{\mathcal{S}}\right]=$ $\left[R^{\dagger}, H_{\mathcal{S}}\right]=0$. That is, translational operators do not evolve in time, so (A12) and (A13) can easily be worked out. In fact, (A12) and (A13) reduce to

$$
\begin{aligned}
H_{\mathrm{eff}}= & H_{\mathcal{S}}-\frac{i(\hbar \Gamma)^{2}}{2 \hbar}\left\{\int_{0}^{\infty} d \tau\left[\chi_{11}(-\tau)-\chi_{11}^{*}(-\tau)\right] R^{\dagger} R\right. \\
& \left.+\int_{0}^{\infty} d \tau\left[\chi_{22}(-\tau)-\chi_{22}^{*}(-\tau)\right] R R^{\dagger}\right\}, \\
F[\bullet]= & \frac{(\hbar \Gamma)^{2}}{\hbar^{2}}\left\{\int_{0}^{\infty} d \tau\left[\chi_{11}(-\tau)+\chi_{11}^{*}(-\tau)\right] R \bullet R^{\dagger}\right. \\
& \left.+\int_{0}^{\infty} d \tau\left[\chi_{22}(-\tau)+\chi_{22}^{*}(-\tau)\right] R^{\dagger} \bullet R\right\} .
\end{aligned}
$$

Here we have used that phonon thermal statistical correlations fulfill $\chi_{\alpha \beta}(-\tau)=0 \forall \alpha \neq \beta$. On the other hand, it is possible to show that at equilibrium

$$
\begin{aligned}
\chi_{11}(-\tau) & =\operatorname{Tr}_{\mathcal{B}}\left[\rho_{\mathcal{B}}^{e q} \mathcal{W}_{1}^{\dagger} \mathcal{W}_{1}(-\tau)\right] \\
& =\sum_{j}\left|v_{j}\right|^{2} e^{i \tau \omega_{j}}\left(e^{\hbar \omega_{j} / k_{B} T}-1\right)^{-1} \\
\chi_{22}(-\tau) & =\operatorname{Tr}_{\mathcal{B}}\left[\rho_{\mathcal{B}}^{e q} \mathcal{W}_{2}^{\dagger} \mathcal{W}_{2}(-\tau)\right] \\
& =\sum_{j}\left|v_{j}\right|^{2} e^{-i \tau \omega_{j}} \frac{e^{\hbar \omega_{j} / k_{B} T}}{e^{\hbar \omega_{j} / k_{B} T}-1}
\end{aligned}
$$

The next step is to adopt an spectral function characterizing the phonon bath. Here we will assume the Ohmic approximation, that is, $\sum_{j}\left|v_{j}\right|^{2} \delta\left(\omega-\omega_{j}\right) \simeq g \omega \Theta\left(\tilde{\omega}_{c}-\omega\right)$, where $g$ is a constant, $\Theta(z)$ the step function, and $\tilde{\omega}_{c}$ the Caldeira-Leggett frequency cutoff. Time integrals appearing in (A14) and (A15) can be computed by invoking half Fourier transforms and Hilbert transforms of correlations; then we get

$$
\begin{aligned}
\int_{0}^{\infty} d \tau\left[\chi_{11}(-\tau)+\chi_{11}^{*}(-\tau)\right] & =\int_{0}^{\infty} d \tau\left[\chi_{22}(-\tau)+\chi_{22}^{*}(-\tau)\right] \\
& =2 \pi g k_{B} T / \hbar \\
\int_{0}^{\infty} d \tau\left[\chi_{11}(-\tau)-\chi_{11}^{*}(-\tau)\right] & =\int_{0}^{\infty} d \tau\left[\chi_{22}(-\tau)-\chi_{22}^{*}(-\tau)\right] \\
& =-i 2 g \tilde{\omega}_{c} .
\end{aligned}
$$

Collecting all these results, the effective Hamiltonian and the superoperator $F[\bullet]$ can be computed, leading to the QME presented in (A11), that is, the KL infinitesimal generator (4) with $D \propto k_{B} T$, as we expected from our thermally activated interaction Hamiltonian model (A2). 
[1] A. E. Allahverdyan, R. Balian, and T. M. Nieuwenhuizen, Phys. Rep. 525, 1 (2013).

[2] H. P. Breuer and F. Petrucione, The Theory of Open Quantum Systems (Oxford University Press, Oxford, 2003).

[3] M. O. Cáceres, Non-Equilibrium Statistical Physics with Application to Disordered Systems (Springer, Berlin, 2017).

[4] R. Alicki and K. Lendi, Quantum Dynamical Semigroups and Applications, Lecture Notes in Physics (Springer, Berlin, 1987), Vol. 286.

[5] E. Joos, H. D. Zeh, C. Kiefer, D. J. W. Giulini, J. Kupsch, and I.-O. Stamatescu, Decoherence and the Appearance of a Classical World in Quantum Theory (Springer, Berlin, 2003).

[6] G. L. Giorgi, F. Plastina, G. Francica, and R. Zambrini, Phys. Rev. A 88, 042115 (2013).

[7] M. Mohseni, P. Rebentrost, S. Lloyd, and A. Aspuru-Guzik, J. Chem. Phys. 129, 174106 (2008).

[8] F. Benatti, F. Carollo, and R. Floreanini, J. Math. Phys. 57, 062208 (2016).

[9] R. Tanaś and Z. Ficek, J. Opt. B 6, S90 (2004).

[10] M. Nizama and M. O. Cáceres, Eur. Phys. J. B 90, 164 (2017).

[11] M. Nizama and M. O. Cáceres, J. Phys. A: Math. Theor. 50, 215208 (2017).

[12] M. O. Cáceres and M. Nizama, J. Phys. A: Math. Theor. 43, 455306 (2010).

[13] M. Nizama and M. O. Cáceres, J. Phys. A: Math. Theor. 45, 335303 (2012).

[14] M. Nizama and M. O. Cáceres, Physica A 392, 6155 (2013).

[15] M. Nizama and M. O. Cáceres, Physica A 400, 31 (2014).
[16] Y. Aharonov, L. Davidovich, and N. Zagury, Phys. Rev. A 48, 1687 (1993).

[17] N. G. van Kampen, J. Stat. Phys. 78, 299 (1995).

[18] M. Nielsen and I. Chuang, Quantum Computation and Quantum Information (Cambridge University Press, Cambridge, 2000).

[19] H. M. Wiseman and J. A. Vaccaro, Phys. Rev. Lett. 91, 097902 (2003).

[20] M. R. Dowling, A. C. Doherty, and H. M. Wiseman, Phys. Rev. A 73, 052323 (2006).

[21] J. Schliemann, J. I. Cirac, M. Kuś, M. Lewenstein, and D. Loss, Phys. Rev. A 64, 022303 (2001).

[22] R. Paškauskas and L. You, Phys. Rev. A 64, 042310 (2001).

[23] P. Zanardi, Phys. Rev. A 65, 042101 (2002).

[24] F. Iemini and R. O. Vianna, Phys. Rev. A 87, 022327 (2013); T. Debarba, R. O. Vianna, and F. Iemini, ibid. 95, 022325 (2017).

[25] H. Shapourian and S. Ryu, Phys. Rev. A 99, 022310 (2019).

[26] A. P. Majtey, C. Zander, and A. R. Plastino, Eur. Phys. J. D 67, 79 (2013); C. Zander and A. R. Plastino, Phys. Rev. A 81, 062128 (2010).

[27] A. Beggi, F. Buscemi, and P. Bordone, Quantum Inf. Process. 15, 3711 (2016).

[28] G. Vidal and R. F. Werner, Phys. Rev. A 65, 032314 (2002).

[29] M. Esposito and P. Gaspard, Phys. Rev. B 71, 214302 (2005).

[30] E. A. Evangelidis, J. Math. Phys. 25, 2151 (1984).

[31] R. Horodecki, P. Horodecki, M. Horodecki, and K. Horodecki, Rev. Mod. Phys. 81, 865 (2009).

[32] K. Modi, A. Brodutch, H. Cable, T. Paterek, and V. Vedral, Rev. Mod. Phys. 84, 1655 (2012). 